TITLE:

\title{
Crystal structure of a thermophilic GrpE protein: insight into thermosensing function for the DnaK chaperone system.
}

AUTHOR(S):

Nakamura, Akira; Takumi, Kouhei; Miki, Kunio

CITATION:

Nakamura, Akira ... [et al]. Crystal structure of a thermophilic GrpE protein: insight into thermosensing function for the DnaK chaperone system.. Journal of molecular biology 2010, 396(4): 1000-1011

\section{ISSUE DATE:}

2010-03-05

URL:

http://hdl.handle.net/2433/132547

\section{RIGHT:}

(C) 2009 Elsevier Ltd; This is not the published version. Please cite only the published version.; この論文は出版社版でありません。引用の際に は出版社版をご確認ご利用ください。 
Title

Crystal structure of a thermophilic GrpE protein: Insight into thermosensing function for the

DnaK chaperone system

\section{Running title}

Structural basis of a thermosensor GrpE

\section{Authors}

Akira Nakamura ${ }^{1}$, Kouhei Takumi ${ }^{1}$, and Kunio Miki ${ }^{1,2^{*}}$

\section{Affiliations}

${ }^{1}$ Department of Chemistry, Graduate School of Science, Kyoto University, Sakyo-ku, Kyoto 606-8502, Japan, ${ }^{2}$ RIKEN SPring-8 Center at Harima Institute, Koto 1-1-1, Sayo, Hyogo 679-5148, Japan

\section{Corresponding author}

*Correspondence should be addressed to Kunio Miki.

Tel: +81-75-753-4029; Fax: +81-75-753-4032; e-mail: miki@kuchem.kyoto-u.ac.jp

Postal Address: Department of Chemistry, Graduate School of Science, Kyoto University, Sakyo-ku, Kyoto 606-8502, Japan 


\section{Abstract}

A homodimeric GrpE protein functions as a nucleotide exchange factor of the eubacterium DnaK molecular chaperone system. The co-chaperone GrpE accelerates ADP dissociation from, and promotes ATP binding to, DnaK, which cooperatively facilitates the DnaK chaperone cycle with another co-chaperone, DnaJ. GrpE characteristically undergoes two-step conformational changes in response to elevation of the environmental temperature. In the first transition at heat-shock temperatures, fully reversible and functionally deficient structural alteration takes place in GrpE, and then the higher temperatures lead to irreversible dissociation of the GrpE dimer into monomers as the second transition. GrpE is also thought to be a thermosensor of the DnaK system, since it is the only member of the DnaK system that changes its structure reversibly and loses its function at heat-shock temperatures of various organisms. We here report the crystal structure of GrpE from Thermus thermophilus HB8 $\left(\mathrm{GrpE}_{T t h}\right)$ at $3.23 \AA$ resolution. The resolved structure is compared with that of GrpE from mesophilic Escherichia coli $\left(\mathrm{GrpE}_{E c o}\right)$, revealing structural similarities, particularly in the DnaK interaction regions, and structural characteristics for the thermal stability of GrpE $\mathrm{E}_{T t h}$. In addition, the structure analysis raised the possibility that the polypeptide chain in the reported $\mathrm{GrpE}_{E c o}$ structure was misinterpreted. Comparison of these two GrpE structures combined with the results of limited proteolysis experiments provide insight into the protein dynamics of $\mathrm{GrpE}_{T t h}$ correlated with the shift of temperature, and also suggest that the 
localized and partial unfolding at the plausible DnaK interaction sites of $\mathrm{GrpE}_{T \text { th }}$ causes functional deficiency of nucleotide exchange factor in response to the heat shock.

\section{Keywords}

unfolding, heat-shock response, thermosensor, thermal stability, protein dynamics

\section{Abbreviations}

nucleotide exchange factor, NEF

DnaK from Escherichia coli, DnaK ${ }_{E c o}$

GrpE from Escherichia coli, GrpE $E_{E c o}$

nucleotide-binding domain, NBD

differential scanning calorimetry, DSC

GrpE from Thermus thermophilus HB8, $\mathrm{GrpE}_{T t h}$

DnaK from Thermus thermophilus HB8, DnaK ${ }_{T t h}$

single-wavelength anomalous dispersion, SAD

metalloendopeptidase, MEP

matrix-assisted laser desorption/ionization, MALDI

mass spectrometry, MS 


\section{Introduction}

Molecular chaperone proteins are involved in various cellular functions, such as protein folding, refolding/degradation of nonnative proteins, prevention of protein aggregation under stress conditions, regulation of protein activities, and so on. ${ }^{1}$ Hsp70 is a ubiquitous molecular chaperone family found in eubacteria, eukarya, and some archaea. Hsp70-family proteins play important roles in the maintenance of protein quality, including folding of nascent polypeptides, transmembrane transport of proteins, and suppression of protein aggregation. ${ }^{2}$ Hsp70 is an ATP-dependent molecular chaperone protein that exhibits weak intrinsic ATPase activity, and it is usually present in an ATP-bound state. Although Hsp70 has low affinity with fast exchange rates for substrates in its ATP-bound state, ADP-bound Hsp70 undergoes an internal conformational change that results in comparatively high affinity with slow exchange rates for substrates. ${ }^{3}$ This structural alteration of Hsp70 is coupled to ATP hydrolysis and ADP/ATP exchange, which is closely related to the actions of the co-chaperones, J-protein (Hsp40) and nucleotide exchange factor (NEF). ${ }^{3}$ Both cooperative proteins are essential for efficient progression of the Hsp70 chaperone cycle.

In prokaryotes, DnaK of Escherichia coli $\left(\mathrm{DnaK}_{E c o}\right)$ is a well-known Hsp70 homologue whose ATPase activity is facilitated by an Hsp40 homologue DnaJ in synergy with substrate binding. ${ }^{2}$ ADP dissociation from DnaK $E_{E c o}$ is accelerated 5000 -fold by an NEF, GrpE, ${ }^{4}$ resulting in efficient ATP rebinding to and substrate release from DnaK. ${ }^{5}$ These three proteins 
constitute the DnaK chaperone system. The crystal structure revealed a direct interaction between the homodimeric GrpE from E. coli $\left(\mathrm{GrpE}_{E c o}\right)$ and the nucleotide-binding domain of DnaK $_{E c o}{ }^{6}$ suggesting a forced opening of the nucleotide-binding cleft of DnaK ${ }_{E c o}$ by insertion of the $\beta$-sheet domain of $\mathrm{GrpE}_{E c o}$. Interestingly, the results of the $\mathrm{CD}$ measurement and differential scanning calorimetry (DSC) indicated that $\mathrm{GrpE}_{E c o}$ is the only component of the DnaK $K_{E c o}$ system that undergoes temperature-dependent conformational changes in the physiologically relevant temperature range, exhibiting two distinctive thermal transitions with midpoints at $\sim 48^{\circ} \mathrm{C}$ and $75-80^{\circ} \mathrm{C}^{7}$ Conformational changes of $\mathrm{GrpE}_{E c o}$ at the lower-temperature transition are fully reversible, and its NEF activity reaches a maximum at $40^{\circ} \mathrm{C}^{7}$ while the second transition represents irreversible inactivation of $\mathrm{GrpE}_{E c o}$. Thus, GrpE $_{E c o}$ becomes transiently unable to interact with the nucleotide-binding domain of DnaK $_{E c o}$ at heat-shock temperatures, resulting in stabilization of the DnaK-ADP-substrate complex. GrpE is therefore thought to act as a thermosensor of the DnaK system that prevents substrate proteins from thermal denaturation. ${ }^{8}$

In addition to $\mathrm{GrpE}_{E c o}$, GrpE of the thermophilic eubacterium Thermus thermophilus HB8 $\left(\mathrm{GrpE}_{T t h}\right)$ has been well characterized, revealing that the two GrpE show similar biochemical and biophysical properties. ${ }^{9}$ The amino acid sequence alignment and CD spectra indicated that these two GrpE proteins probably have common structural features, including the domain composition, i.e., the N-terminal long $\alpha$-helix, four-helix bundle, and C-terminal $\beta$-sheet 
domains. $^{10}$ GrpE $_{\text {Tth }}$ binds to DnaK from T. thermophilus HB8 (DnaK Tth ) in a 2:1 stoichiometry and accelerates ADP/ATP exchange of DnaK ${ }_{T t h}$ up to 80,000 fold, ${ }^{10}$ although DnaJ $\mathrm{J}_{T \text { th }}$ does not affect ATP hydrolysis of DnaK ${ }_{T t h}{ }^{10,11}$ forming a large $300 \mathrm{kDa}$ complex composed of trimers of DnaK $\mathrm{Tth}_{T h}$, DnaJ $\mathrm{J}_{T t h}$, and the assembly factor DafA. ${ }^{12}$ Further, the two-step thermal transition observed in the DSC measurement has suggested that $\mathrm{GrpE}_{T t h}$ has the ability to work as a thermosensor of the DnaK ${ }_{T t h}$ system. ${ }^{8}$ Intriguingly, $\mathrm{GrpE}_{T t h}$ can heterologously interact with DnaK $K_{E c o}$ and accelerate the nucleotide release from DnaK $K_{E c o}$ with less efficiency than the homologous complexes, $\mathrm{GrpE}_{T t h}-\mathrm{DnaK}_{T t h}$ and $\mathrm{GrpE}_{E c o}-\mathrm{DnaK} \mathrm{K}_{E c o}{ }^{10}$ despite the low-level sequence identity between the GrpE proteins from these two species (26\%). Therefore, it has been expected that the fundamental mechanism of the nucleotide exchange reaction for DnaK by GrpE is sufficiently conserved between the mesophilic and thermophilic DnaK systems. ${ }^{10}$

While GrpE $E_{E c o}$ and $\mathrm{GrpE}_{T t h}$ have functional similarity, as described above, they also show significant differences in thermal stability and temperature-dependent conformational changes, which are tightly correlated with the optimal growth temperature of each organism. The first part of the temperature-induced structural transition of $\mathrm{GrpE}_{E c o}$ has been attributed to the unfolding of the N-terminal long paired helices, ${ }^{13}$ which is linked to the destabilization of the $\beta$-sheet domain. ${ }^{14}$ In the case of $\mathrm{GrpE}_{T t h}$, the lower-temperature transition with a midpoint at $90^{\circ} \mathrm{C}$ has been alternatively ascribed to the unfolding of the C-terminal $\beta$-sheet 
domain, not the $\mathrm{N}$-terminal helices. ${ }^{8}$ Meanwhile, the second transition (at $75-80^{\circ} \mathrm{C}$ in $\operatorname{GrpE}_{E c o}{ }^{7}$ and at $100-105^{\circ} \mathrm{C}$ in $\mathrm{GrpE}_{T t h}{ }^{8}$ ) has been considered to be responsible for the irreversible dissociation of the GrpE dimer in both species. ${ }^{8,13,14}$ Structural information on $\mathrm{GrpE}_{T t h}$ will therefore play a key role in informing the discussion about its thermostability and the structural mechanisms involved in its functions. Here we report the crystal structure of $\mathrm{GrpE}_{\text {Tth }}$ and a novel structural basis for its thermosensing action. 


\section{Results and Discussion}

\section{Overall structure of $\mathrm{GrpE}_{T t h}$ and comparison with $\mathrm{GrpE}_{E c o}$}

The crystal structure of $\mathrm{GrpE}_{\text {Tth }}$ expressed in E. coli was determined at $3.23 \AA$ resolution using the single-wavelength anomalous dispersion (SAD) technique (Figure 1a). Surprisingly, despite the large cell volume $\left(\sim 210^{3} \AA^{3}\right)$, only two GrpE $E_{T h h}$ molecules forming a homodimer exist in a crystallographic asymmetric unit with a high solvent content (87\%). The GrpE $\mathrm{T}_{\text {Tth }}$ dimer adopts a flat and sea angel (clione)-like shape composed of three domains in a protomer (the N-terminal long $\alpha$-helix, central four-helix bundle, and C-terminal $\beta$-sheet domains), as found in the structure of $\operatorname{GrpE}_{E c o}$ (Figure $\left.1 \mathrm{~b}\right) .{ }^{6}$ The structural similarity between these two GrpE proteins expected previously ${ }^{8,10}$ was corroborated by the results of this study as follows (see Figures 1 and 2).

Superimposition of each domain structure between two GrpE structures results in rmsd values of $3.7 \AA(47 \mathrm{C} \alpha), 1.1 \AA$ (41 C $\alpha)$, and $1.5 \AA$ (55 C $\alpha$ ) for $\mathrm{C} \alpha$ atoms of the long $\alpha$-helix, four-helix bundle, and $\beta$-sheet domains, respectively (Figure 1d). In this superimposition, the topological conversion for $\mathrm{GrpE}_{E c o}$, described in the next section in detail, was taken into consideration. These values for the structural comparison were closely correlated with the degree of amino acid sequence identity (22\%, 33\%, and 31\%, respectively). As the C-terminal $\beta$-sheet domain of $\mathrm{GrpE}_{E c o}$ is mainly responsible for the interaction with DnaK $_{E c o}{ }^{6}$ the remarkably similar shape of the $\beta$-sheet domain of these two GrpE proteins is 
thought to contribute to the functional complementarity of $\mathrm{GrpE}_{T t h}$ with the heterogeneous DnaK $_{\text {Eco }}$ system. $^{10}$

While the long $\alpha$-helix domain of $\mathrm{GrpE}_{E c o}$ did not form a canonical coiled-coil structure and the N-terminal 33 residues were disordered in the crystal structure, ${ }^{6}$ the typical coiled-coil was observed in the corresponding region of $\mathrm{GrpE}_{T t h}$. A major reason for this difference may have been the sequence diversity of the long $\alpha$-helix domain, which is supported by the lower sequence identity of this domain (22\%) compared to that of the other domains. GrpE $\mathrm{E}_{\text {Tth }}$ has the almost complete $\mathrm{i}+3, \mathrm{i}+4$ heptad repeat of hydrophobic residues from the N-terminus, and the N-terminal coiled-coil is also predicted by the COILS server, ${ }^{15}$ whereas $\mathrm{GrpE}_{E c o}$ contains an imperfect amino acid sequence for the coiled-coil formation (Figure 2).

The $\alpha$-helix domain of $\operatorname{GrpE}_{T t h}$ has a relatively higher mean $B$-factor value $\left(\sim 198 \AA^{2}\right)$ than that of the other domains $\left(\sim 73 \AA^{2}\right)$, suggesting the structural flexibility of this domain. Despite the conformational fluctuation, we could construct the $\mathrm{GrpE}_{T t h}$ model from the vicinity of the $\mathrm{N}$-termini because the $\mathrm{N}$-terminal 20 residues of the $\mathrm{GrpE}_{\text {Tth }}$ dimer contact other N-termini of $\mathrm{GrpE}_{\text {Tth }}$ molecules from an adjacent crystallographic asymmetric unit and the conformations of the $\mathrm{N}$-terminal regions are therefore stabilized to some extent each other in the crystal. 


\section{Comparison of topology between $\operatorname{GrpE}_{T t h}$ and $\mathrm{GrpE}_{E c o}$}

Although both the GrpE $E_{T t h}$ and $\mathrm{GrpE}_{E c o}$ dimers have similar overall structures, we found a difference in the protein topology between the two dimer formations. For the sake of clarity, we designated the long $\alpha$-helix including $\alpha 1$ and $\alpha 2$ (Figure 2) as $\alpha A\left(\alpha A^{\prime}\right), \alpha 3$ as $\alpha B\left(\alpha B^{\prime}\right)$, and the $\beta$-sheet domain as $\beta$ ( $\beta^{\prime}$ ) (see diagrams in Figure 1 ). As for the $\mathrm{GrpE}_{E c o}$ structure from the database (PDB ID: $\underline{\mathbf{1 D K G}}$ ), a linker inside of the four-helix bundle domain connects $\alpha A^{\prime}$ and $\alpha B$ (or $\alpha A$ and $\alpha B^{\prime}$ ), resulting in an $\alpha A^{\prime}-\alpha B-\beta$ ( $\left.\alpha A^{-}-\alpha B^{\prime}-\beta^{\prime}\right)$ linkage (Figure 1b). Therefore, the two subunits of the $\mathrm{GrpE}_{E c o}$ dimer were classified as protomers proximal and distal to DnaK $E_{E c o}$ that bound to $\mathrm{GrpE}_{E c o}$ in a $1: 2$ stoichiometry (Figure 1b). ${ }^{6}$ This classification was made by Harrison et al., who built their $\mathrm{GrpE}_{E c o}$ model based on the discontinuous and weak electron density at the linker region in the four-helix bundle domain. ${ }^{6}$ In contrast to this $\mathrm{GrpE}_{E c o}$ model (here designated topology $\mathrm{A}$ ), the corresponding linker of $\operatorname{GrpE}_{T t h}$ alternatively connects $\alpha \mathrm{A}$ and $\alpha \mathrm{B}$ ( $\alpha \mathrm{A}^{\prime}$ and $\alpha \mathrm{B}^{\prime}$; here designated topology $\mathrm{B}$ ) (Figure 1a), which is unambiguously supported by the explicitly observed experimental electron density in this region (Figure 3). Thus, we investigated whether the previously reported linker connectivity of $\mathrm{GrpE}_{E c o}{ }^{6}$ was reasonable from a structural viewpoint. The dimerization surface is mainly constructed of the four-helix bundle domains in both quaternary structures, and $4400 \AA^{2}$ or $5800 \AA^{2}$ of surface area is buried upon dimer formation in topologies A and B, respectively. Furthermore, the distributions of hydrophobic residues and electrostatic 
potential maps also indicate that topology B can lead to stronger intersubunit interaction than topology A (Figure 4 and Table 1). Since a previously reported DSC experiment for GrpE $E_{E c o}$ in which the temperature of the $\operatorname{GrpE}_{E c o}$ solution was elevated beyond the normal growth conditions showed that the unfolding of the long $\alpha$-helix domain precedes the dissociation of the $\mathrm{GrpE}_{E c o}$ dimer, ${ }^{13}$ strong interactions at the dimer interface are considered to be necessary for conforming with the biological properties. In all cases, topology B has more favorable structural features than topology A. Therefore, topology B is suggested to be the correct conformation for $\mathrm{GrpE}_{E c o}$, as seen in $\mathrm{GrpE}_{T t h}$, and a modified $\mathrm{GrpE}_{E c o}$ structure adopting topology B (here designated as $\mathrm{Grp}_{E c o}{ }^{*}$ ), in which the chain assignments for the 116-197 residues were interconverted to their counterparts, was hereinafter used as the GrpE $E_{E c o}$ model. We think that the poor electron density of the $\mathrm{GrpE}_{E c o}$ linker ${ }^{6}$ is insufficient for proper linkage of the polypeptide chains. However, we cannot completely exclude the possibility that topology $\mathrm{A}$ is the adequate conformation for $\mathrm{GrpE}_{E c o}$, and that GrpE proteins have structural diversity in their functional shapes, as suggested previously. ${ }^{16}$

\section{Thermal stability of GrpE $E_{T h}$}

The optimal growth temperature of $T$. thermophilus $\left(70-75^{\circ} \mathrm{C}\right)$ is at least $30^{\circ}$ higher than that of E. coli $\left(37^{\circ} \mathrm{C}\right)$. Comparison of the amino acid composition reveals that $\mathrm{GrpE}_{T t h}$ has essential features to acquire enhanced thermotolerance relative to $\mathrm{GrpE}_{E c o}$ (Table 2). Notably, 
the proportion of charged polar residues of $\operatorname{Grp}_{T t h}(41.2 \%)$ is significantly greater than that of $\mathrm{GrpE}_{E c o}$ (33.5\%), especially in the case of the residues glutamate (17.5\% versus $13.2 \%$ for $\mathrm{GrpE}_{T t h}$ and $\mathrm{GrpE}_{E c o}$, respectively) and arginine (10.7\% versus 5.6\%). Correspondingly, there are fewer unionized polar residues in $\operatorname{GrpE}_{T t h}$ (7.3\%) compared to $\operatorname{GrpE}_{E c o}(16.2 \%)$. In addition, structure analysis of $\mathrm{GrpE}_{T \text { th }}$ also shows evidence for its intrinsic thermostability. The unstructured loop regions inside of the four-helix bundle and $\beta$-sheet domains were shortened by three residues each (Figure 2). The GrpE $E_{\text {Th }}$ dimer buries $6200 \AA^{2}$ of the monomer solvent-accessible surface area, which is significantly larger than that for the $\mathrm{GrpE}_{E c o}$ * dimer $\left(5800 \AA^{2}\right)$. The calculated electrostatic potential maps distinctly indicate that the N-terminal half of the four-helix bundle $(\alpha 2)$ includes a negatively charged patch whereas the C-terminal half is positively charged, and also illustrate that the electrostatically charged area of $\mathrm{GrpE}_{T t h}$ is clearly larger than that of $\mathrm{GrpE}_{E c o} *$ (Figure 5). This electrostatic complementarity in addition to the wide charged area would make it possible to form extensive polar interactions at the dimerization interface of $\mathrm{Grp}_{T t h}$ rather than $\mathrm{GrpE}_{E c o}$. This is supported by the much larger number of intersubunit ionic pairs between the four-helix bundle domains of $\mathrm{GrpE}_{T t h}$ compared to $\mathrm{GrpE}_{E c o}$ * (8 versus 0; Table 1). Further, several intrasubunit ionic pairs inside of the $\mathrm{GrpE}_{T \text { th }} \beta$-sheet domains could serve to increase the conformational stability of this domain even at the relatively high growth temperature of $T$. thermophilus compared to E. coli. All these features generally found in thermophilic 
proteins ${ }^{17-19}$ contribute to the expression of the thermal stability of GrpE $E_{T t h}$.

\section{Localized partial unfolding at heat-shock temperatures}

Far-UV CD spectra of GrpE $E_{T t h}$ taken at two different temperatures, including the normal growth temperature $\left(70^{\circ} \mathrm{C}\right)$ and the heat-shock temperature $\left(90^{\circ} \mathrm{C}\right)$ of $T$. thermophilus, show that there is no statistically significant change upon the temperature shift from $70^{\circ} \mathrm{C}$ to $90^{\circ} \mathrm{C}$ (Figure 6 and Table 3), although the DSC data explicitly suggest a structural transition of $\mathrm{GrpE}_{T \text { th }}$ at $90^{\circ} \mathrm{C} .^{8}$ These results lead us to hypothesize that $\mathrm{GrpE}_{T t h}$ undergoes a structure alteration in the $\beta$-sheet domain, which is an "invisible” domain in CD analysis. ${ }^{8}$ However, with respect to the temperature-induced conformational changes, we were uncertain about the idea that the entire $\beta$-sheet domain of $\mathrm{GrpE}_{T t h}$ unfolds at heat-shock temperatures as suggested previously. ${ }^{8}$ We therefore performed limited proteolysis experiments and subsequent matrix-assisted laser desorption/ionization time-of-flight mass spectrometry (MALDI TOF-MS) analysis for the resultant peptide fragments to investigate how much area of $\mathrm{GrpE}_{\text {Tth }}$ unfolds by the heat shock. A thermophilic protease, metalloendopeptidase (MEP) from Grifola frondosa, that cleaves peptide bonds on the N-terminal side of lysine residues, was used for digestion of $\mathrm{GrpE}_{T \text { th }}$ at room temperature, $70^{\circ} \mathrm{C}$, and $90^{\circ} \mathrm{C}$ with time course incubation. If the whole structure of the $\beta$-sheet domain were disrupted by the heat shock at $90^{\circ} \mathrm{C}$, MEP could attack peptide bonds related to all three lysine residues (Lys143, Lys146, 
and Lys170) in the $\beta$-sheet domain and then the corresponding peptide fragments would be detected as the proteolytic products at $90^{\circ} \mathrm{C}$. However, no mass signals corresponding to fragments that had been digested at the Lys143 position were detected, although MEP cleaved $\mathrm{GrpE}_{T t h}$ at the other two lysine positions (Figure 7 and Table 4). In this case, no bands corresponding to degraded peptides of $\mathrm{GrpE}_{T t h}$ were detected under any of the temperature conditions without addition of MEP (Figure 7a). SDS-PAGE analysis (Figure 7) also shows that GrpE $E_{\text {Tth }}$ was hydrolyzed by MEP at the Gly11-Lys12 bond (i.e. fragment 2 was produced) immediately even at room temperature, and also indicates that fragments 3 (12-169 residues), fragment 5 (12-145), fragment 6 (29-145), and fragment 7 (54-145) with their complementary short peptides (Fragments 9-11) were generated at $90^{\circ} \mathrm{C}$ rapidly and preferentially rather than at $70^{\circ} \mathrm{C}$, although only fragment $4(29-177)$ and a fraction of fragment 5 were detected in the $70^{\circ} \mathrm{C}$ products.

In order to interpret this difference in digestion patterns appropriately, we should consider the effects of the temperature shift on both MEP and GrpE $E_{T t h}$. It is reported that the optimum temperature of MEP is $70^{\circ} \mathrm{C}$ and its activity is decreased to $20 \%$ at $90^{\circ} \mathrm{C}$ for 1 hour. ${ }^{20}$ In addition, we examined temperature-dependent reaction rates of MEP by monitoring degradation of the full-length $\mathrm{GrpE}_{T t h}$ under single-hit conditions, in which the limited proteolysis was performed at a $\mathrm{GrpE}_{T t h} / \mathrm{MEP}$ ratio of $5000: 1$ for $0-120$ min. Because full-length $\mathrm{GrpE}_{T t h}$ is rapidly truncated by MEP at the N-terminal side of Lys12 even at room 
temperature (generation of fragment 2), this area must be structurally flexible at $70^{\circ} \mathrm{C}$ and $90^{\circ} \mathrm{C}$. Our experiments indicate that MEP reaction at $90^{\circ} \mathrm{C}$ is at least three times slower than that at $70^{\circ} \mathrm{C}$ (data not shown). This reduction in MEP activity at $90^{\circ} \mathrm{C}$ is also found in the results of proteolysis under mild conditions $\left(\mathrm{GrpE}_{T t h} / \mathrm{MEP}=1000: 1\right)$, in which full-length $\mathrm{GrpE}_{\text {Tth }}$ significantly remains after 10 min of incubation with $\mathrm{MEP}$ at $90^{\circ} \mathrm{C}$ rather than at $70^{\circ} \mathrm{C}$ (Figure 7a). However, fragments 3, 5, 6, and 7 are distinctively observed even in the 10 -min reaction product at $90^{\circ} \mathrm{C}$ and the generation of these fragments is obviously faster than that at $70^{\circ} \mathrm{C}$. These results imply that the remarkable difference in MEP digestion patterns between $70^{\circ} \mathrm{C}$ and $90^{\circ} \mathrm{C}$ reflects a temperature-dependent conformational change of $\mathrm{GrpE}_{T \text { th }}$ that is not due to increased activation of MEP. Thus, we believe that the structure of the regions around Lys54, Lys146, and Lys170 would be easily disrupted in response to increasing temperature to the extent that MEP could attack their peptide bonds. The fact that structural changes at $90^{\circ} \mathrm{C}$ are induced mainly in the $\beta$-sheet domain is consistent with the finding from our CD measurement. On the other hand, MEP cannot hydrolyze the Gly142-Lys143 bond either at $70^{\circ} \mathrm{C}$ or at $90^{\circ} \mathrm{C}$, suggesting that the area around Lys143 retain its three-dimensional structure at $90^{\circ} \mathrm{C}$ and has comparatively high thermoresistance. Considering these findings, we can deduce that the structural characteristics of $\mathrm{GrpE}_{T t h}$ are as follows. (1) A dozen N-terminal residues do not adopt a particular conformation even at room temperature in aqueous solution. (2) At heat-shock temperatures, the localized partial 
unfolding takes place around Lys54, Lys146, and Lys170, whereas the region around Lys143 keeps its conformation.

\section{Correlation between the localized unfolding and functional deficiency of NEF}

The region essential for the thermosensing action of $\mathrm{GrpE}_{E c o}$ is assigned to within the $\alpha$-helix domain, ${ }^{13}$ and the unfolding of this sensor domain induces destabilization of the $\beta$-sheet domain conformation at heat-shock temperatures through linkages between the two domains, e.g., a cation- $\pi$ interaction between Phe86 and Arg183. ${ }^{14}$ Despite the functional and structural similarities between $\mathrm{GrpE}_{T t h}$ and $\mathrm{GrpE}_{E c o}$, similar interactions between the helical domains and $\beta$-sheet domain were not observed in our $\mathrm{GrpE}_{T t h}$ crystal structure, implying that these domains of $\mathrm{GrpE}_{T t h}$ are thermodynamically independent. Moreover, the N-terminal long $\alpha$-helix domain of $\mathrm{GrpE}_{T t h}$ forms a stable coiled-coil, as described above, suggesting that this region is no longer a thermosensor, unlike $\mathrm{GrpE}_{E c o}$. In other words, the intrinsic structural mechanisms for the heat-shock response of the two GrpE proteins seem to be different. To understand the thermosensing mechanism of $\mathrm{GrpE}_{T t h}$, we investigated the relationship between the localized unfolding and functional deficiency of NEF. On the basis of the conformational resemblance of GrpE proteins and a high degree of sequence identity (65\%) in DnaK proteins from T. thermophilus and E. coli, it is thought that $\mathrm{Grp}_{T \text { th }}$ associates with DnaK $_{T t h}$ in a manner similar to that seen in the crystal structure of the $\mathrm{GrpE}_{E c o}-\mathrm{DnaK}_{E c o}$ 
complex; ${ }^{6}$ that is, DnaK $\mathrm{K}_{\text {th }}$ makes contact with $\mathrm{GrpE}_{\text {Tth }}$ from one side (designated as the "front" side) of the GrpE dimer (Figure 8), and interaction sites are mainly found in the $\beta$-sheet domain with narrow interspersed areas in the other domains (Figures 2 and $8 \mathrm{~b}$ ). Amino acid residues in this contact face of GrpE are better conserved than those on the other side (the "back" side) between GrpE $\mathrm{E}_{T \text { th }}$ and $\mathrm{GrpE}_{E c o}$ (Figures 8b and 8c). In addition, the temperature-sensitive Lys54, Lys146, and Lys170, indicated by the limited proteolysis experiment, are located on this DnaK interaction interface (Figure 8d). In contrast, Lys143, at which $\mathrm{GrpE}_{T t h}$ was not cleaved by MEP even at $90^{\circ} \mathrm{C}$, forms an ion pair with Glu140 at the interface opposite the DnaK interaction interface, and all of the intrasubunit ionic pairs found in the $\beta$-sheet domain (Table 1 ) are located on this rigid back side (Figure $8 \mathrm{~d}$ ). Due to the postulated localized unfolding that arose in the DnaK interaction region by the heat shock, $\mathrm{GrpE}_{\text {Tth }}$ might be structurally inadequate to interact with $\mathrm{DnaK}_{T t h}$ and to open the nucleotide-binding pocket of $\mathrm{DnaK}_{T t h}$ for release of ADP. Therefore, it appears that GrpE $\mathrm{E}_{T t h}$ has an insufficient structural environment to function as NEF for DnaK $\mathrm{K}_{T \text { th }}$ at heat-shock temperatures. Consequently, we conclude that the localized and partial unfolding of $\mathrm{Grp}_{\mathrm{Tth}}$, which is elicited by the heat shock and occurred at the contact area of DnaK $\mathrm{K}_{\text {Tth }}$, is a structural characteristic for the low-temperature transition in DSC and is also a structural basis for the functional deficiency of NEF at heat-shock temperatures. By virtue of the site-specified and small-scale unfolding, $\mathrm{GrpE}_{\text {Tth }}$ would effectively become an inactive form for the nucleotide 
exchange at heat-shock temperatures and it could be refolded and reactivated as NEF at permissive temperatures, implying that the NEF-inactivation in response to the heat shock would be structurally and functionally reversible (Figure 9). 


\section{Methods}

\section{Cloning, expression, and purification}

The grpE gene (TTHA1490) from T. thermophilus HB8 was cloned into the NdeI/BamHI sites of pET-11a (Novagen, Darmstadt, Germany), and the recombinant plasmid was transformed into E. coli strain BL21(DE3). Cells were grown at $37^{\circ} \mathrm{C}$ in $\mathrm{LB}$ medium containing $100 \mu \mathrm{g} / \mathrm{ml}$ ampicillin, and the expression of the $\mathrm{GrpE}_{T t h}$ protein was performed without addition of isopropyl $\beta$-D-thiogalactopyranoside. The harvested cells were resuspended and sonicated in lysis buffer $(20 \mathrm{mM}$ Tris- $\mathrm{HCl}, 50 \mathrm{mM} \mathrm{NaCl}, 5 \mathrm{mM}$ $\beta$-mercaptoethanol, $\mathrm{pH} 8.0$ ). The soluble lysate was incubated at $70^{\circ} \mathrm{C}$ for $10 \mathrm{~min}$, cooled on ice, and centrifuged at $200,000 \times g$ for $60 \mathrm{~min}$ at $4^{\circ} \mathrm{C}$. The $\mathrm{GrpE}_{T t h}$ protein was precipitated in lysis buffer containing $1.5 \mathrm{M}$ ammonium sulfate. The precipitant was then isolated by centrifugation and resuspended in the lysis buffer. The solution containing $\mathrm{GrpE}_{T \text { th }}$ was applied to a Resource Q column (GE Healthcare Biosciences, Tokyo, Japan) equilibrated with $20 \mathrm{mM}$ Tris-HCl (pH 8.0) and then eluted with a linear gradient of $\mathrm{NaCl}$. The GrpE $_{T t h}$-containing fraction was applied to a hydroxyapatite column (Bio-Scale CHT10-I; Bio-Rad, Tokyo, Japan) equilibrated with $10 \mathrm{mM}$ sodium phosphate and $150 \mathrm{mM} \mathrm{NaCl}(\mathrm{pH}$ 7.0) and then eluted with a sodium phosphate gradient. $\mathrm{GrpE}_{T t h}$ was applied to a Hi-Load 16/60 Superdex 75 pg column (GE Healthcare Biosciences) equilibrated with $20 \mathrm{mM}$ Tris- $\mathrm{HCl}$ and $150 \mathrm{mM} \mathrm{NaCl}(\mathrm{pH}$ 8.0) and then eluted with the same buffer. The purified 
$\mathrm{GrpE}_{\text {Tth }}$ solution was desalted and concentrated to $28.8 \mathrm{mg} / \mathrm{ml}$ in $20 \mathrm{mM}$ Tris- $\mathrm{HCl}$ and $1 \mathrm{mM}$ DTT (pH 8.0).

\section{Crystallization, data collection, and structure determination}

Crystals of $\mathrm{GrpE}_{T t h}$ were obtained by the hanging-drop vapor diffusion method at $20^{\circ} \mathrm{C}$.

Drops of $\mathrm{GrpE}_{\text {Tth }}$ were mixed with an equal volume of the reservoir solution containing 100

$\mathrm{mM}$ sodium acetate $\left(\mathrm{pH}\right.$ 5.0) and $700 \mathrm{mM} \mathrm{MgSO}_{4}$. For structure determination of $\mathrm{GrpE}_{T t h}$, isomorphous heavy-atom derivative crystals of $\mathrm{Pt}$ and Au were prepared by soaking in reservoir solutions containing $10 \mathrm{mM}$ potassium tetrachloroplatinate for $5 \mathrm{~h}$ and $10 \mathrm{mM}$ potassium dicyanoaurate for $24 \mathrm{~h}$, respectively. X-ray diffraction data were collected using an ADSC Quantum-315 detector on the BL41XU beamline at SPring-8 (Native1, Pt, and Au). Crystals were soaked in the reservoir solution containing 25\% $(v / v)$ ethylene glycol and then flash-frozen in a liquid nitrogen stream at $100 \mathrm{~K}$ before data collection. Native crystals for higher-resolution data were grown under almost the same crystallization conditions described above, except for addition of acetone at a final concentration of 1.5\% $(v / v)$ to the reservoir solution. X-ray diffraction data of these crystals were collected using an ADSC Quantum-210 detector on the NW12 beamline at PF-AR (Native2). All of the data sets were processed with the HKL-2000 program package. ${ }^{21}$ Initial phases were calculated using Pt and Au derivative data sets by the SAD method using SOLVE. ${ }^{22}$ Three Pt and Au sites were successfully determined, and an interpretable electron density map was obtained. The phase probability 
distributions from derivative data sets were combined using the SIGMAA program of $\mathrm{CCP} 4,{ }^{23}$ and then density modification was performed by $\mathrm{RESOLVE}^{24}$ together with the native data set (Native1) at $3.75 \AA$ resolution. Two GrpE $E_{T h}$ molecules were found in an asymmetric unit with a Matthews coefficient $V_{\mathrm{M}}$ value ${ }^{25}$ of $9.7 \AA^{3} / \mathrm{Da}$, corresponding to a solvent content of $87.3 \%$. Several cycles of manual model building and computational refinement were carried out using the programs $\mathrm{COOT}^{26}$ and $\mathrm{CNS},{ }^{27,28}$ respectively, until the crystallographic $R$-factor equaled $23.4 \%\left(R_{\text {free }}=24.2 \%\right)$ at $3.52 \AA$ resolution. Atomic coordinates of the refined structure (Native1) were used as a model of the molecular replacement method with the program Molrep ${ }^{29}$ to determine the phase angles of the structural factors of the Native2 crystal that gave better X-ray diffraction than the Native1 crystal. The GrpE $\mathrm{E}_{\text {Tth }}$ structure was refined to the crystallographic $R$ and $R_{\text {free }}$ factors of $21.8 \%$ and $23.8 \%$, respectively, at $3.23 \AA$ resolution. The final model was validated using the program MolProbity. ${ }^{30}$ The statistics are summarized in Table 5. All discussion of GrpE $_{T t h}$ in this paper is based on the structure of the Native2 crystal unless otherwise noted. All figures representing GrpE structures were prepared with PyMOL. ${ }^{31}$

\section{CD spectroscopy}

Far-UV CD spectra were recorded with a J-805 spectropolarimeter (JASCO, Tokyo, Japan) from $250 \mathrm{~nm}$ to $190 \mathrm{~nm}$ at a scan rate of $50 \mathrm{~nm} / \mathrm{min}, 1 \mathrm{~nm}$ resolution, $1 \mathrm{~nm}$ bandwidth, a time constant of $1.0 \mathrm{~s}$, a sensitivity of $100 \mathrm{mdeg}$, and an accumulation of 10 scans. The 
protein concentration was $2.5 \mu \mathrm{M}$ in $20 \mathrm{mM}$ sodium phosphate (pH 8.0), and the cuvette path length was $2 \mathrm{~mm}$. Spectra of the identical $\mathrm{GrpE}_{T t h}$ solution were measured at two discrete temperature conditions $\left(70^{\circ} \mathrm{C}\right.$ and $\left.90^{\circ} \mathrm{C}\right)$, in which the temperature was held constant using a water bath. The secondary structure content against the mean residue ellipticity at 190-240 nm was estimated by means of the reference set by Yang. ${ }^{32}$

\section{Limited proteolysis experiments}

Limited proteolysis experiments were performed in $20 \mathrm{mM}$ Tris- $\mathrm{HCl}(\mathrm{pH} 8.0)$ by incubating $\operatorname{GrpE}_{T t h}(50 \mu \mathrm{M})$ with metalloendopeptidase (MEP) from G. frondosa (Seikagaku Biobusiness Corp., Tokyo, Japan) at a GrpE $\mathrm{T}_{T h} / \mathrm{MEP}$ ratio of 5000:1, 1000:1, or 500:1 (w/w). Enzymatic digestions were carried out for $0-120$ min or $1-3 \mathrm{~h}$ at room temperature, $70^{\circ} \mathrm{C}$, or $90^{\circ} \mathrm{C}$, and were terminated by freezing. The digestion patterns were analyzed by SDS-PAGE.

\section{Mass spectroscopy}

A variety of MEP-digested $\mathrm{GrpE}_{T t h}$ solutions were diluted 5-fold by $0.1 \%(\mathrm{v} / \mathrm{v})$ trifluoroacetic acid, and a $2-\mu \mathrm{l}$ portion of each diluted sample was mixed with an equal volume of a matrix solution containing $10 \mathrm{mg} / \mathrm{ml}$ sinapinic acid, 50\% (v/v) acetonitrile, and $0.05 \%(v / v)$ trifluoroacetic acid. Two microliters of the mixtures were spotted onto a MALDI target plate and allowed to dry at room temperature (dried-droplet method). Mass spectra were acquired using a Voyager-DE PRO Biospectrometry Workstation (Applied Biosystems, Foster City, CA) in linear mode with external calibration using a ProteoMass Protein 
MALDI-MS Calibration Kit (Sigma-Aldrich, St. Louis, MO).

\section{Accession number}

Coordinates and structure factors for $\mathrm{GrpE}_{T t h}$ have been deposited in the PDB under accession code $\underline{\mathbf{3 A 6 M}}$. 


\section{Acknowledgements}

We thank the beamline scientists of SPring-8 (BL41XU) and of the Photon Factory (NW12) for their kind help in the X-ray diffraction experiments. This work was performed as part of the "Structurome" Project of the RIKEN Harima Institute, and the initial preparation of protein samples carried out by Prof. S. Kuramitsu and his coworkers is greatly appreciated. 


\section{References}

1. Hartl, F. U. \& Hayer-Hartl, M. (2002). Molecular chaperones in the cytosol: from nascent chain to folded protein. Science 295, 1852-1858.

2. Bukau, B., Weissman, J. \& Horwich, A. (2006). Molecular chaperones and protein quality control. Cell 125, 443-451.

3. Bukau, B. \& Horwich, A. L. (1998). The Hsp70 and Hsp60 chaperone machines. Cell 92, 351-366.

4. Packschies, L., Theyssen, H., Buchberger, A., Bukau, B., Goody, R. S. \& Reinstein, J. (1997). GrpE accelerates nucleotide exchange of the molecular chaperone DnaK with an associative displacement mechanism. Biochemistry 36, 3417-3422.

5. Mally, A. \& Witt, S. N. (2001). GrpE accelerates peptide binding and release from the high affinity state of DnaK. Nat. Struct. Biol. 8, 254-257.

6. Harrison, C. J., Hayer-Hartl, M., Di Liberto, M., Hartl, F. \& Kuriyan, J. (1997). Crystal structure of the nucleotide exchange factor GrpE bound to the ATPase domain of the molecular chaperone DnaK. Science 276, 431-435.

7. Grimshaw, J. P., Jelesarov, I., Schonfeld, H. J. \& Christen, P. (2001). Reversible thermal transition in GrpE, the nucleotide exchange factor of the DnaK heat-shock system. J. Biol. Chem. 276, 6098-6104.

8. Groemping, Y. \& Reinstein, J. (2001). Folding properties of the nucleotide exchange factor GrpE from Thermus thermophilus: GrpE is a thermosensor that mediates heat shock response. J. Mol. Biol. 314, 167-178.

9. Schlee, S. \& Reinstein, J. (2002). The DnaK/ClpB chaperone system from Thermus thermophilus. Cell. Mol. Life Sci. 59, 1598-1606.

10. Groemping, Y., Klostermeier, D., Herrmann, C., Veit, T., Seidel, R. \& Reinstein, J. (2001). Regulation of ATPase and chaperone cycle of DnaK from Thermus thermophilus by the nucleotide exchange factor GrpE. J. Mol. Biol. 305, 1173-1183.

11. Klostermeier, D., Seidel, R. \& Reinstein, J. (1999). The functional cycle and regulation of the Thermus thermophilus DnaK chaperone system. J. Mol. Biol. 287, 511-525.

12. Motohashi, K., Yohda, M., Endo, I. \& Yoshida, M. (1996). A novel factor required for the assembly of the DnaK and DnaJ chaperones of Thermus thermophilus. J. Biol. Chem. 271, 17343-17348.

13. Gelinas, A. D., Langsetmo, K., Toth, J., Bethoney, K. A., Stafford, W. F. \& Harrison, C. J. (2002). A structure-based interpretation of E.coli GrpE thermodynamic properties. J. Mol. Biol. 323, 131-142.

14. Gelinas, A. D., Toth, J., Bethoney, K. A., Langsetmo, K., Stafford, W. F. \& Harrison, C. J. (2003). Thermodynamic linkage in the GrpE nucleotide exchange factor, a 
molecular thermosensor. Biochemistry 42, 9050-9059.

15. Lupas, A., Van Dyke, M. \& Stock, J. (1991). Predicting coiled coils from protein sequences. Science 252, 1162-1164.

16. Harrison, C. (2003). GrpE, a nucleotide exchange factor for DnaK. Cell Stress Chaperones 8, 218-224.

17. Vieille, C. \& Zeikus, G. J. (2001). Hyperthermophilic enzymes: sources, uses, and molecular mechanisms for thermostability. Microbiol. Mol. Biol. Rev. 65, 1-43.

18. Tanaka, T., Sawano, M., Ogasahara, K., Sakaguchi, Y., Bagautdinov, B., Katoh, E., Kuroishi, C., Shinkai, A., Yokoyama, S. \& Yutani, K. (2006). Hyper-thermostability of CutA1 protein, with a denaturation temperature of nearly 150 degrees C. FEBS Lett. 580, 4224-4230.

19. Matsui, I. \& Harata, K. (2007). Implication for buried polar contacts and ion pairs in hyperthermostable enzymes. FEBS J. 274, 4012-4022.

20. Nonaka, T., Ishikawa, H., Tsumuraya, Y., Hashimoto, Y. \& Dohmae, N. (1995). Characterization of a thermostable lysine-specific metalloendopeptidase from the fruiting bodies of a basidiomycete, Grifola frondosa. J. Biochem. 118, 1014-1020.

21. Otwinowski, Z. \& Minor, W. (1997). Processing of X-ray diffraction data collected in oscillation mode. Methods Enzymol. 276, 307-326.

22. Terwilliger, T. C. \& Berendzen, J. (1999). Automated MAD and MIR structure solution. Acta Crystallogr., Sect. D: Biol. Crystallogr. 55, 849-861.

23. Collaborative Computational Project, Number 4. (1994). The CCP4 suite: programs for protein crystallography. Acta Crystallogr., Sect. D: Biol. Crystallogr. 50, 760-763.

24. Terwilliger, T. C. (2000). Maximum-likelihood density modification. Acta Crystallogr., Sect. D: Biol. Crystallogr. 56, 965-972.

25. Matthews, B. W. (1968). Solvent content of protein crystals. J. Mol. Biol. 33, 491-497.

26. Emsley, P. \& Cowtan, K. (2004). Coot: model-building tools for molecular graphics. Acta Crystallogr., Sect. D: Biol. Crystallogr. 60, 2126-2132.

27. Brünger, A. T., Adams, P. D., Clore, G. M., DeLano, W. L., Gros, P., Grosse-Kunstleve, R. W., Jiang, J. S., Kuszewski, J., Nilges, M., Pannu, N. S., Read, R. J., Rice, L. M., Simonson, T. \& Warren, G. L. (1998). Crystallography \& NMR system: A new software suite for macromolecular structure determination. Acta Crystallogr., Sect. D: Biol. Crystallogr. 54, 905-921.

28. Brunger, A. T. (2007). Version 1.2 of the Crystallography and NMR system. Nat. Protoc. 2, 2728-2733.

29. Vagin, A. \& Teplyakov, A. (1997). MOLREP: an Automated Program for Molecular Replacement. J. Appl. Crystallogr. 30, 1022-1025.

30. Davis, I. W., Leaver-Fay, A., Chen, V. B., Block, J. N., Kapral, G. J., Wang, X., 
Murray, L. W., Arendall, W. B., 3rd, Snoeyink, J., Richardson, J. S. \& Richardson, D. C. (2007). MolProbity: all-atom contacts and structure validation for proteins and nucleic acids. Nucleic Acids Res. 35, W375-383.

31. DeLano, W. L. (2002). The PyMOL Molecular Graphics System, DeLano Scientific, Palo Alto, CA.

32. Yang, J. T., Wu, C. S. \& Martinez, H. M. (1986). Calculation of protein conformation from circular dichroism. Methods Enzymol. 130, 208-269.

33. Kabsch, W. \& Sander, C. (1983). Dictionary of protein secondary structure: pattern recognition of hydrogen-bonded and geometrical features. Biopolymers 22, 2577-2637. 


\section{Figure captions}

Figure 1.

Structural comparisons of $\mathrm{Grp}_{\text {Tth }}$ with $\mathrm{GrpE}_{E c o}$. Overall architectures of (a) $\mathrm{GrpE}_{\text {Tth }}$ and (b)

$\mathrm{GrpE}_{E c o}$ (extracted from the PDB entry, ID: 1DKG; topology A) are illustrated in a ribbon representation with several colors. Top views of these models are shown below. The position of DnaK interacting with the proximal GrpE $E_{E c o}$ molecule (magenta) is indicated by the dotted circle in (b). Bottom cartoons illustrate the polypeptide-chain connectivity of GrpE proteins. The $\alpha$ A ( $\alpha A^{\prime}$ )-labeled circle indicates the $\alpha$-helix domain $(\alpha 1)$ and the first $\alpha$-helix of the four-helix bundle domain ( $\alpha 2)$. The $\alpha \mathrm{B}$ ( $\alpha \mathrm{B}^{\prime}$ )-labeled circle represents the second $\alpha$-helix of the four-helix bundle domain ( $\alpha 3)$. The $\beta$ ( $\beta^{\prime}$ )-labeled rectangle depicts the $\beta$-sheet domain. (c) $\mathrm{GrpE}_{E c o}$ in the topology $\mathrm{B}\left(\mathrm{GrpE}_{E c o}{ }^{*}\right)$ constructed by the authors as a modification of $\mathrm{GrpE}_{E c o}$ is shown. The polypeptide chain assignment is altered and the new chains A and B are colored magenta and red, respectively. The elements of the bottom cartoon are the same as in (a) and (b). (d) Superimpositions of the domain structures are shown. GrpE $E_{\text {Tth }}$ models are colored in blue and cyan, and $\mathrm{GrpE}_{E c o} *$ models are colored in red and magenta.

Figure 2.

Amino acid sequence alignment of the GrpE proteins. Identical and homologous residues are colored green and orange, respectively. The secondary structure elements of $\mathrm{GrpE}_{T \text { th }}$ and 
$\mathrm{GrpE}_{E c o}$ based on their crystal structures are illustrated above and below the sequences, respectively. Blue triangles indicate the characteristic MEP cleavage sites of $\mathrm{GrpE}_{T t h}$ at $90^{\circ} \mathrm{C}$. $\mathrm{GrpE}_{E c o}$ residues interacting with $\mathrm{DnaK}_{E c o}$ in the crystal structure of their complex are represented with red triangles.

Figure 3.

A stereo view of the initial experimental electron density map around the linker region of the four-helix bundle domain. The map is overlaid on the final $\mathrm{GrpE}_{T t h}$ model (Native1). The color assignments are the same as Figure 1a.

Figure 4.

Comparison of the two possible topologies for the $\mathrm{GrpE}_{E c o}$ structure. (a) Topology A, the original form of the PDB entry. (b) Topology B, the modified form as observed in the crystal structure of GrpE $E_{T t h}$. Simplified diagrams, hydrophobic regions on the molecular surface, and electrostatic potential maps are drawn from the left panel, respectively. The blue and red colors on the electrostatic potential maps indicate regions of positive potential $(>+5 \mathrm{kT} / \mathrm{e})$ and of negative potential ( $<-5 \mathrm{kT} / \mathrm{e})$ values, respectively. Only chain A, which corresponds to the magenta model in the left panel, is shown in the central and right panels. 
Figure 5.

Comparison of the electrostatic interaction on the GrpE dimerization interfaces. Electrostatic potential maps overlaid on one subunit of $\mathrm{GrpE}_{T t h}$ and $\mathrm{GrpE}_{E c o} *$ (topology B) are shown. The contour level was $\pm 5 \mathrm{kT} / \mathrm{e}$ (the same as in Figure 4). The left cartoon illustrates the strong electrostatic complementarities observed on the dimerization surface of $\mathrm{GrpE}_{T t h}$. The first-half helix of the four-helix bundle domain is charged negatively, and the second half is positive.

Figure 6.

Far-UV CD spectra of GrpE $E_{T t h}$. The CD spectra were measured at $70^{\circ} \mathrm{C}$ (continuous line), and $90^{\circ} \mathrm{C}$ (dotted line). Secondary structure contents from the CD spectra and from the crystal structure were deduced using the reference set of Yang $^{32}$ and using the DSSP $^{33}$ program, respectively, and their values are summarized in Table 3.

Figure 7.

Digestion patterns of $\mathrm{GrpE}_{T \text { th }}$ cleaved by metalloendopeptidase (MEP). (a) Limited proteolysis experiments were performed at a $\mathrm{GrpE}_{T t h} / \mathrm{MEP}$ ratio of $1000: 1$ for $0-120$ min at $70^{\circ} \mathrm{C}$ or $90^{\circ} \mathrm{C}$. (b) $\mathrm{GrpE}_{\text {Tth }}$ was digested with MEP (500:1) for 1-3 hours. MEP-cleavage products of $\mathrm{GrpE}_{T t h}$ were separated by 18\% SDS-PAGE and stained with Coomassie brilliant 
blue. $\mathrm{M}$ represents molecular weight markers. Clear bands numbered on the gel for the reaction product were identified by TOF-MS analysis and the results are summarized in Table 4.

Figure 8.

Structural features of the interaction with DnaK. (a) $\mathrm{GrpE}_{E c o}$ (white, surface model) interacts with DnaK $E_{E c o}$ (cyan, ribbon model) in the crystal structure, ${ }^{6}$ and the red area (the residues indicated by red triangles in Figure 2) of $\mathrm{GrpE}_{E c o}$ is related to DnaK-binding. (b) The corresponding $\mathrm{GrpE}_{T t h}$ residues are also colored red. (c) Identical and homologous amino acid residues between $\mathrm{GrpE}_{T t h}$ and $\mathrm{GrpE}_{E c o}$ are indicated in green and orange on the $\mathrm{GrpE}_{T t h}$ structure, respectively, as in Figure 2. Residues of the expected DnaK-binding side are better conserved than those of the other side. (d) Structural features around the expected DnaK-binding region of $\mathrm{GrpE}_{T t h}$. Expected DnaK-binding residues of GrpE $\mathrm{E}_{T \text { th }}$ are colored red. MEP-sensitive residues (magenta, K146 and K170; red, K54) are located at or around the expected DnaK-binding area ("front” side). The predicted DnaK position is indicated by the dashed circle. Three ion pairs (yellow) were found on the distal side ("back" side) of the DnaK-binding region.

Figure 9. 
Model for the localized and partial unfolding of $\mathrm{GrpE}_{T t h}$. During the first transition at $90^{\circ} \mathrm{C}$, the DnaK-binding regions in the $\beta$-sheet and $\alpha$-helix domains unfold reversibly, resulting in the loss of the NEF function. The locations of the lysine residues inside the temperature-sensitive regions are indicated. This figure was made by arranging Groemping's model. $^{8}$ 
Table 1. The distribution of the ionic pairs and hydrogen bonds

\begin{tabular}{|c|c|c|c|}
\hline & $\mathrm{GrpE}_{T t h}$ & GrpE $_{E c o}$ & $\mathrm{GrpE}_{E c o}{ }^{*}$ \\
\hline \multicolumn{4}{|l|}{ Ionic pairs $(<5 \AA)$} \\
\hline Inter-subunit & 28 & 6 & 24 \\
\hline$\alpha$-helix : $\alpha$-helix & 3 & 5 & 5 \\
\hline$\alpha$-helix : four-helix & 2 & 0 & 0 \\
\hline four-helix : four-helix & 8 & 2 & 0 \\
\hline four-helix : $\beta$-sheet & 15 & 0 & 19 \\
\hline Intra-subunit (chain A/B) & $16 / 20$ & $21 / 24$ & $15 / 13$ \\
\hline$\alpha$-helix : $\alpha$-helix & $2 / 4$ & $7 / 8$ & $7 / 8$ \\
\hline four-helix : four-helix & $8 / 4$ & $2 / 2$ & $4 / 2$ \\
\hline four-helix : $\beta$-sheet & $0 / 0$ & $12 / 14$ & $4 / 3$ \\
\hline$\beta$-sheet : $\beta$-sheet & $6 / 12$ & $0 / 0$ & $0 / 0$ \\
\hline \multicolumn{4}{|l|}{ Hydrogen bonds (<3.3 $\AA$ ) } \\
\hline Inter-subunit & 5 & 2 & 1 \\
\hline$\alpha$-helix : $\beta$-sheet & 1 & 0 & 1 \\
\hline four-helix : four-helix & 0 & 2 & 0 \\
\hline four-helix : $\beta$-sheet & 4 & 0 & 0 \\
\hline Intra-subunit (chain A/B) & $118 / 113$ & $125 / 124$ & $123 / 127$ \\
\hline$\alpha$-helix : $\alpha$-helix & $36 / 30$ & $52 / 53$ & $52 / 53$ \\
\hline$\alpha$-helix : four-helix & $5 / 4$ & $2 / 4$ & $2 / 4$ \\
\hline$\alpha$-helix : $\beta$-sheet & $0 / 0$ & $1 / 0$ & $0 / 0$ \\
\hline four-helix : four-helix & $41 / 45$ & 35/36 & $38 / 35$ \\
\hline four-helix : $\beta$-sheet & $2 / 2$ & $1 / 1$ & $1 / 1$ \\
\hline$\beta$-sheet : $\beta$-sheet & $34 / 32$ & $34 / 30$ & $30 / 34$ \\
\hline
\end{tabular}

The Asp residue at the G122D substitution of GrpE $E_{E c o}$ (PDB ID: 1DKG) was replaced by the Gly residue, in which the atoms of the side chain had been removed.

The water molecules were omitted in the calculations for the number of the hydrogen bonds. 
Table 2. The amino acid composition of GrpE proteins

\begin{tabular}{|c|c|c|c|c|c|}
\hline & \multicolumn{2}{|l|}{$\mathrm{GpE}_{T \text { th }}$} & \multicolumn{2}{|l|}{$\mathrm{GrpE}_{E c o}$} & \multirow{2}{*}{$\begin{array}{l}\text { Ratio of a.a. composition } \\
\text { in \% }\left(\mathrm{Grp}_{T t h} / \mathrm{GrpE}_{E c o}\right)\end{array}$} \\
\hline & Residues & $(\%)$ & Residues & $(\%)$ & \\
\hline Charged polar & 73 & 41.24 & 66 & 33.50 & 1.23 \\
\hline Asp & 10 & 5.65 & 13 & 6.60 & 0.86 \\
\hline Glu & 31 & 17.51 & 26 & 13.20 & 1.33 \\
\hline Lys & 11 & 6.21 & 13 & 6.60 & 0.94 \\
\hline Arg & 19 & 10.73 & 11 & 5.58 & 1.92 \\
\hline His & 2 & 1.13 & 3 & 1.52 & 0.74 \\
\hline Uncharged polar & 13 & 7.34 & 32 & 16.24 & 0.45 \\
\hline Ser & 2 & 1.13 & 7 & 3.55 & 0.32 \\
\hline Thr & 1 & 0.56 & 8 & 4.06 & 0.14 \\
\hline Asn & 3 & 1.69 & 8 & 4.06 & 0.42 \\
\hline Gln & 4 & 2.26 & 8 & 4.06 & 0.56 \\
\hline Туг & 3 & 1.69 & 1 & 0.51 & 3.34 \\
\hline Nonpolar & 91 & 51.41 & 99 & 50.25 & 1.02 \\
\hline Gly & 15 & 8.47 & 8 & 4.06 & 2.09 \\
\hline Ala & 21 & 11.86 & 24 & 12.18 & 0.97 \\
\hline Val & 12 & 6.78 & 17 & 8.63 & 0.79 \\
\hline Leu & 23 & 12.99 & 16 & 8.12 & 1.60 \\
\hline Ile & 3 & 1.69 & 13 & 6.60 & 0.26 \\
\hline Met & 3 & 1.69 & 9 & 4.57 & 0.37 \\
\hline Phe & 7 & 3.95 & 3 & 1.52 & 2.60 \\
\hline Pro & 7 & 3.95 & 9 & 4.57 & 0.87 \\
\hline Total & 177 & 100 & 197 & 100 & \\
\hline
\end{tabular}


Table 3. The secondary structure fractions of $\mathrm{GrpE}_{T \text { th }}$

\begin{tabular}{crrc}
\hline & $70{ }^{\circ} \mathrm{C}$ & $90^{\circ} \mathrm{C}$ & $\begin{array}{c}\text { crystal } \\
\text { structure }\end{array}$ \\
\hline$\alpha$-helix & $48.3 \%$ & $48.0 \%$ & $55.9 \%$ \\
$\beta$-strand & $13.6 \%$ & $13.4 \%$ & $16.1 \%$ \\
turn & $6.7 \%$ & $7.2 \%$ & $5.1 \%$ \\
random & $31.4 \%$ & $31.4 \%$ & $22.9 \%$ \\
\hline RMS & 6.336 & 7.154 & - \\
\hline
\end{tabular}


Table 4. Identification of the peptide fragments from the limited proteolysis

\begin{tabular}{cccc}
\hline Fragments & Peptide & Measured mass & $\begin{array}{c}\text { Expected mass } \\
{[\mathrm{M}+\mathrm{H}]^{+}}\end{array}$ \\
\hline 1 & $1-177$ & $20,024.7 \pm 0.0$ & $20,027.7$ \\
2 & $12-177$ & $18,643.2 \pm 5.1$ & $18,644.2$ \\
3 & $12-169$ & $17,672.8 \pm 4.9$ & $17,673.0$ \\
4 & $29-177$ & $16,762.3 \pm 4.6$ & $16,763.2$ \\
5 & $12-145$ & $14,989.4 \pm 4.0$ & $14,988.0$ \\
6 & $29-145$ & $13,108.8 \pm 3.5$ & $13,107.0$ \\
7 & $54-145$ & $10,126.6 \pm 3.0$ & $10,125.6$ \\
8 & $61-145$ & $9,209.0 \pm 2.1$ & $9,209.5$ \\
9 & $146-177$ & $3,676.2 \pm 1.9$ & $3,675.2$ \\
$10 \mathrm{a}$ & $29-53$ & $2,998.6 \pm 3.9$ & $3,000.4$ \\
$10 \mathrm{~b}$ & $146-169$ & $2,703.5 \pm 4.1$ & $2,704.2$ \\
11 & $12-28$ & $1,900.0 \pm 4.0$ & $1,900.1$ \\
\hline
\end{tabular}


Table 5. Data collection and refinement statistics

\begin{tabular}{|c|c|c|c|c|}
\hline & Native1 & $\mathrm{Pt}$ & $\mathrm{Au}$ & Native2 \\
\hline \multicolumn{5}{|l|}{ Data collection } \\
\hline Space group & $P 4_{3} 32$ & $P 4_{3} 32$ & $P 4_{3} 32$ & $P 4_{3} 32$ \\
\hline \multicolumn{5}{|l|}{ Cell dimensions } \\
\hline$a(\AA)$ & 208.6 & 209.3 & 208.8 & 210.4 \\
\hline X-ray source & SPring-8 BL41XU & SPring-8 BL41XU & SPring-8 BL41XU & PF AR NW12 \\
\hline Wavelength ( $\AA$ ) & 1.0000 & 1.0719 & 1.0000 & 1.0000 \\
\hline Resolution $(\AA)^{*}$ & $50-3.52(3.65-3.52)$ & $50-3.75(3.88-3.75)$ & $50-3.83(3.97-3.83)$ & $50-3.23(3.35-3.23)$ \\
\hline Total reflections & 420,060 & 352,483 & 653,532 & 375,571 \\
\hline Unique reflections & 19,822 & 16,684 & 15,559 & 26,058 \\
\hline Completeness (\%) & $100.0(100.0)$ & $100.0(100.0)$ & $100.0(100.0)$ & $99.8(100.0)$ \\
\hline$<I>/<\sigma(I)>^{*}$ & $37.8(11.3)$ & $38.3(9.2)$ & $44.6(15.7)$ & $54.4(10.8)$ \\
\hline$R_{\mathrm{sym}}{ }^{*, \dagger}$ & $0.090(0.356)$ & $0.121(0.352)$ & $0.123(0.343)$ & $0.059(0.307)$ \\
\hline Phasing & & SAD & SAD & \\
\hline Resolution $(\AA)$ & $50-3.75$ & $50-3.81$ & $50-3.90$ & \\
\hline FOM after SOLVE & & 0.30 & 0.26 & \\
\hline FOM after RESOLVE & 0.73 & & & \\
\hline \multicolumn{5}{|l|}{ Refinement } \\
\hline Resolution ( $\AA$ ) & & & & $36.62-3.23$ \\
\hline$R_{\text {work }} / R_{\text {free }}{ }^{\ddagger}$ & & & & $0.218 / 0.238$ \\
\hline \multicolumn{5}{|l|}{ No. of atoms } \\
\hline Protein / Water & & & & 2,449 / 18 \\
\hline \multicolumn{5}{|l|}{ Average $B$-factors } \\
\hline Protein / Water $\left(\AA^{2}\right)$ & & & & $118.7 / 72.6$ \\
\hline Rmsd bond length ( $\AA$ ) & & & & 0.007 \\
\hline Rmsd bond angles $\left(^{\circ}\right)$ & & & & 1.3 \\
\hline \multicolumn{5}{|l|}{ Ramachandran analysis" } \\
\hline Favored (\%) & & & & 95.1 \\
\hline Outliers (\%) & & & & 0.3 \\
\hline
\end{tabular}

*Values in parentheses are for the highest resolution shell.

${ }^{\dagger} R_{\text {sym }}=\Sigma_{h k l} \Sigma_{i}\left|I_{i}(h k l)-<I(h k l)>\right| / \Sigma_{h k l} \Sigma_{i} I_{i}(h k l)$, where $I_{i}(h k l)$ is the ith intensity measurement of reflection $h k l$ and $\langle I(h k l)>$ is the mean intensity obtained from multiple observations of symmetry-related reflections.

${ }^{\ddagger} R_{\text {work }}=\Sigma|| F_{\text {obs }}|-| F_{\text {calc }} \| / \Sigma\left|F_{\text {obs }}\right| . R_{\text {free }}$ is the same as $R_{\text {work }}$, but for $5 \%$ subset of all reflections that were never used in crystallographic refinement.

"Calculated by MolProbity. 
(a)
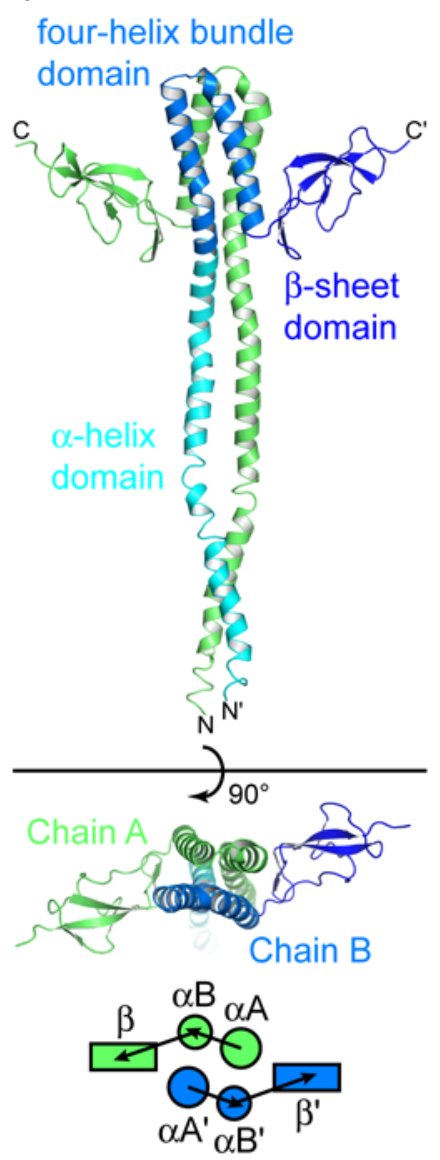

$\operatorname{GrpE}_{\text {Th }}$

(d)

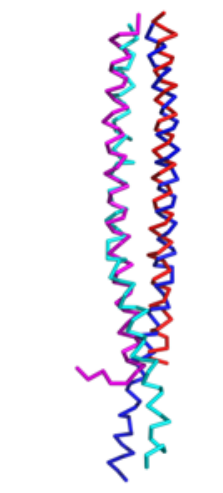

$\alpha$-helix domain (b)
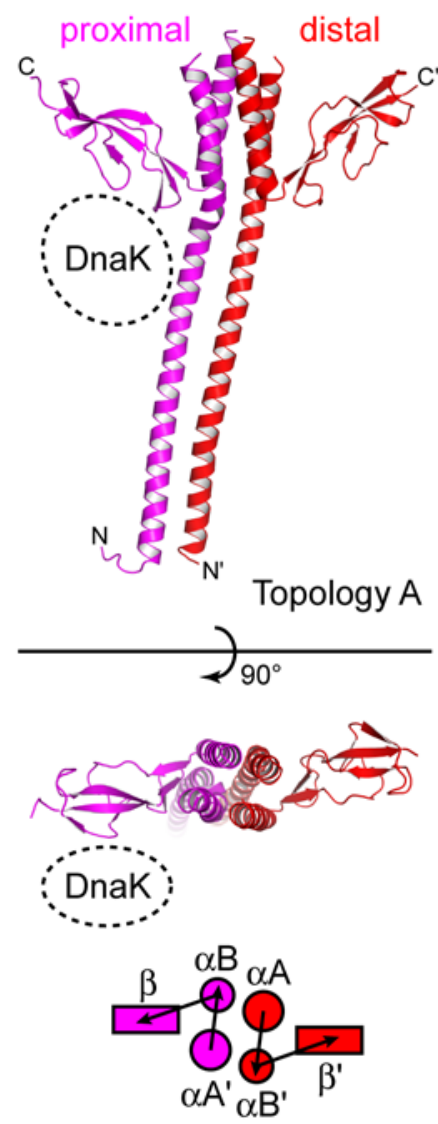

$\operatorname{GrpE}_{E c o}(1 \mathrm{DKG})$

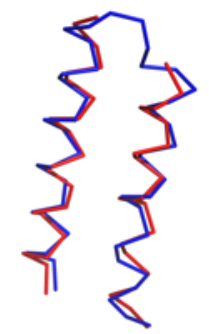

four-helix bundle domain (c)
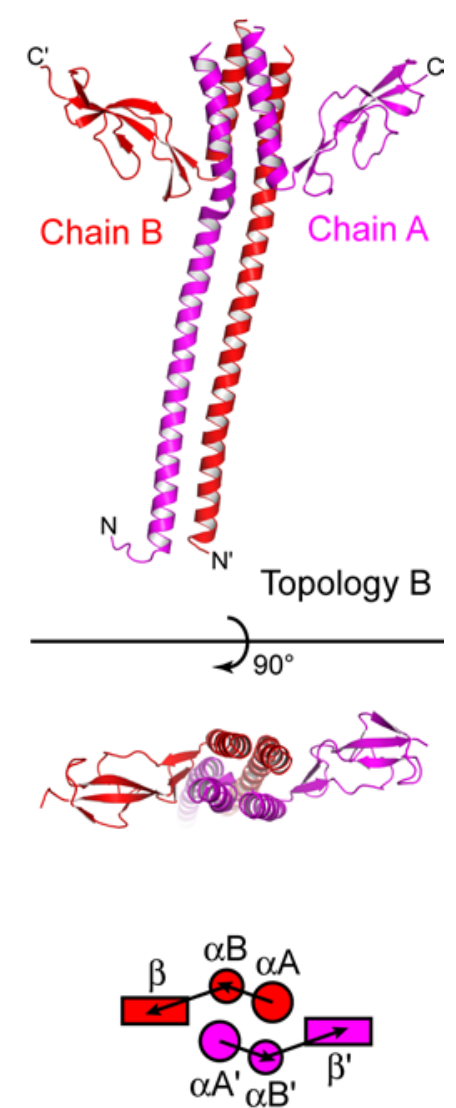

$\operatorname{GrpE}_{E c o}{ }^{*}$ (Topology B)

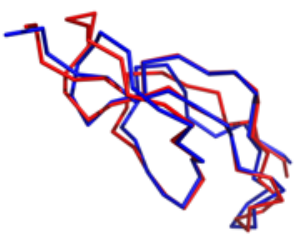

$\beta$-sheet domain

Figure 1 Nakamura et al. 


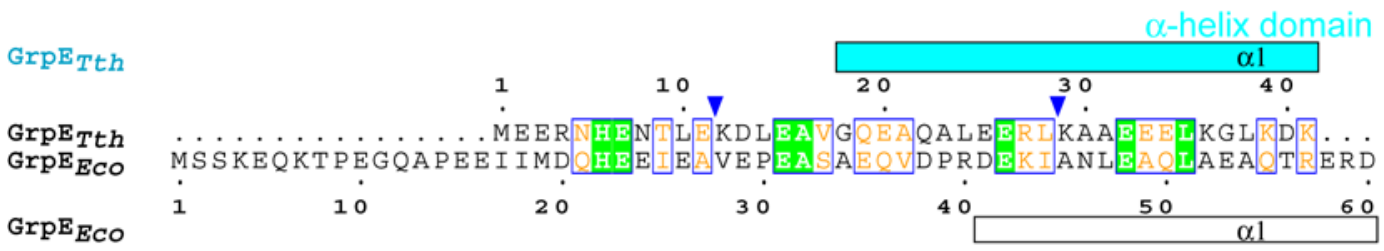

$\operatorname{GrpE}_{T t h}$

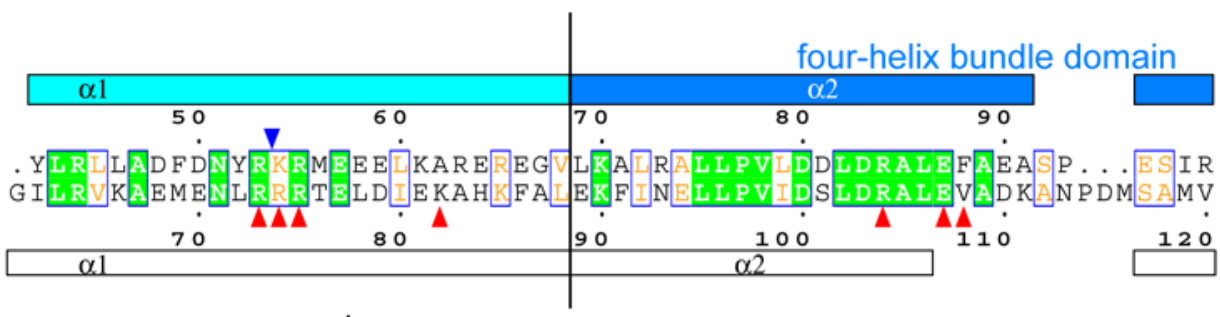

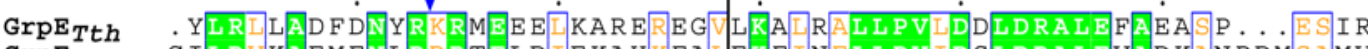

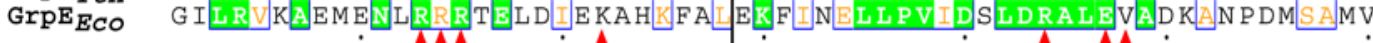
$\operatorname{GrpE}$

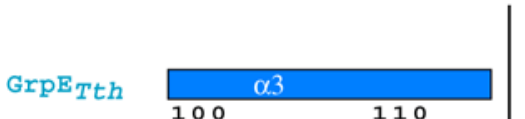
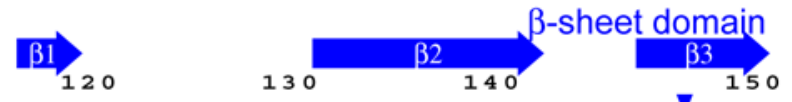

$\operatorname{GrpE}_{T t h}$
$\operatorname{GrpE}_{E C O}$

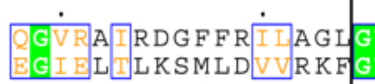

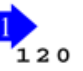

$130 \quad 140$ $\nabla$

$\operatorname{GrpE}_{E C O}$
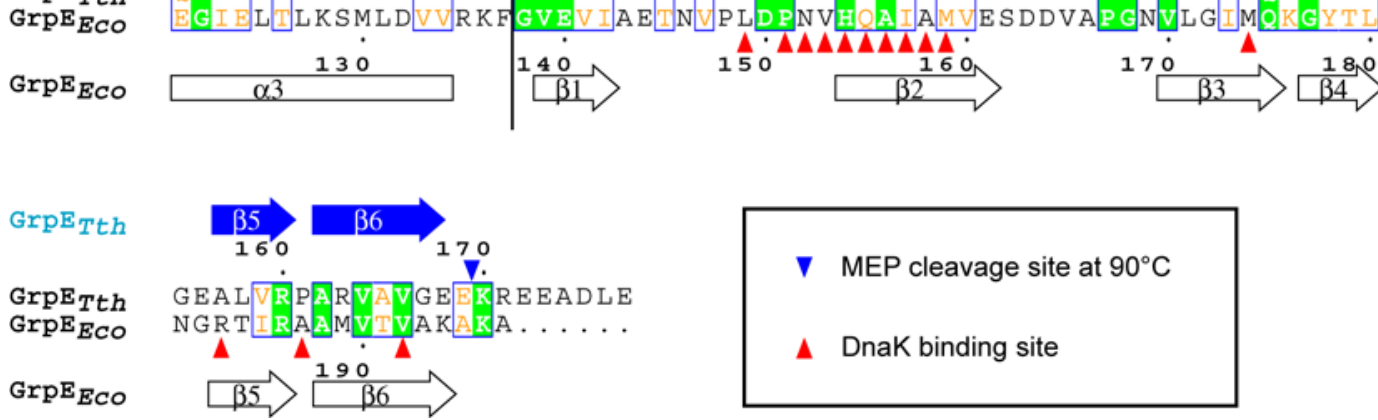

$\nabla$ MEP cleavage site at $90^{\circ} \mathrm{C}$

A DnaK binding site

Figure 2 Nakamura et al. 


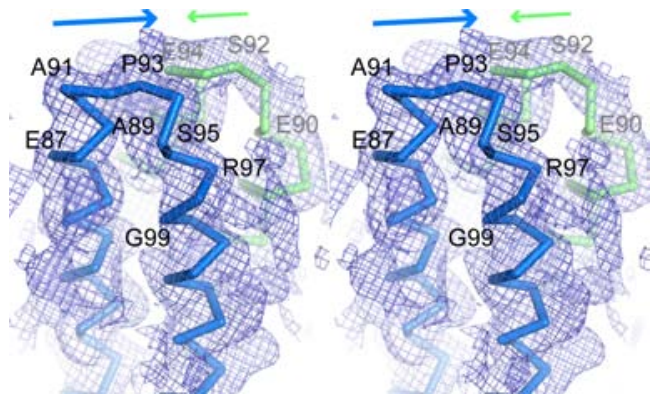

Figure 3 Nakamura et al. 
(a) Topology $\mathrm{A}$ $\left(\mathrm{GrpE}_{E c o}\right)$

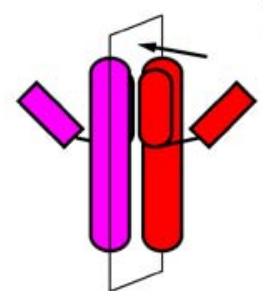

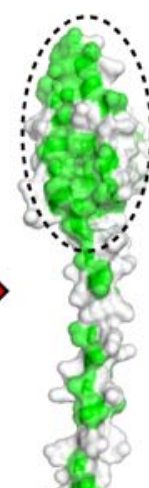

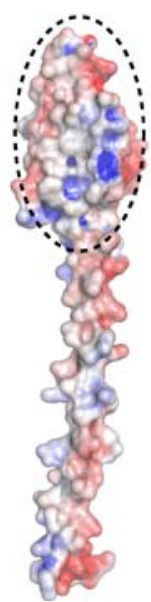

(b) Topology B
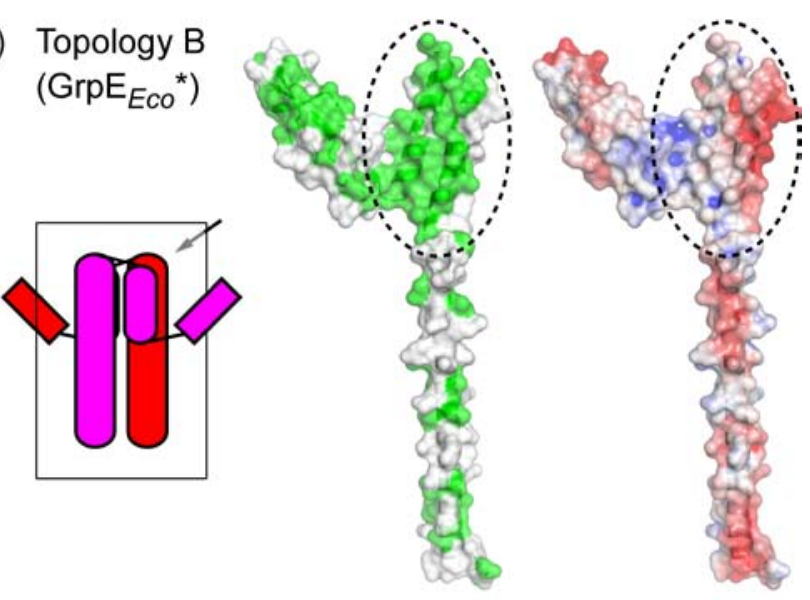

Figure 4 Nakamura et al. 


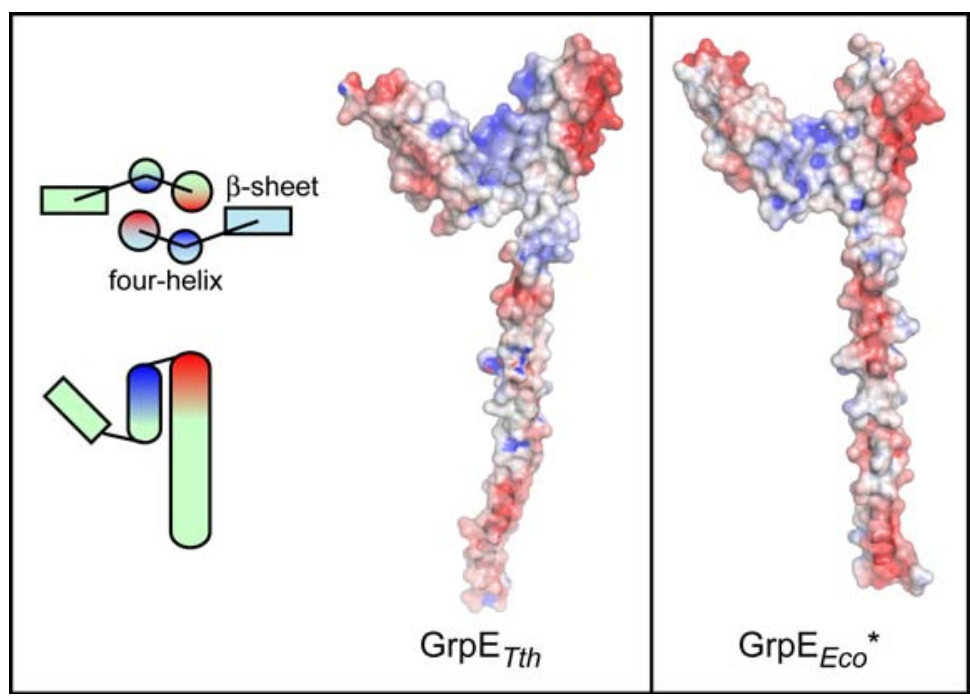

Figure 5 Nakamura et al. 


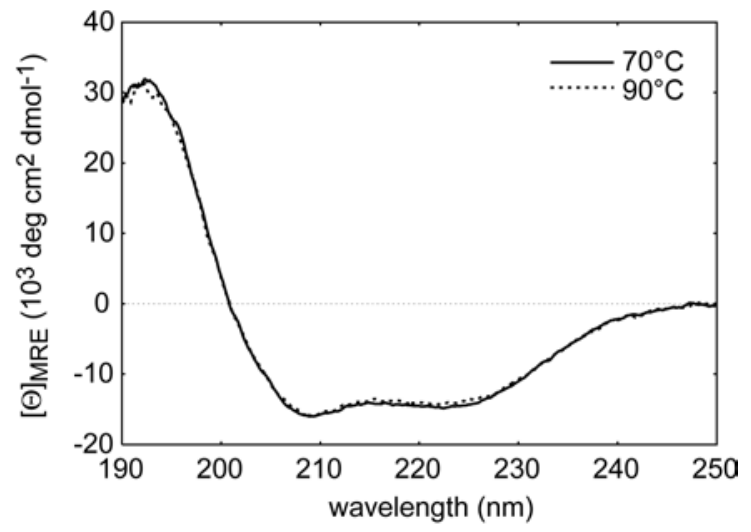

Figure 6 Nakamura et al. 
(a) $\mathrm{GrpE}_{T \text { th }}: \mathrm{MEP}=1000: 1$

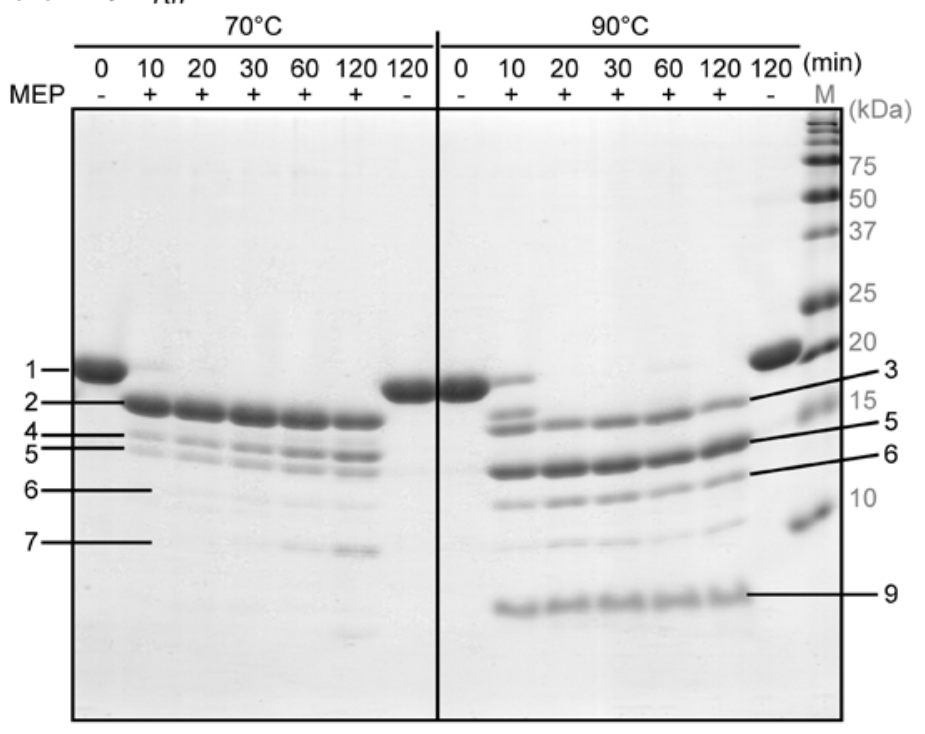

(b) $\operatorname{GrpE}_{T t h}$ :MEP $=500: 1$

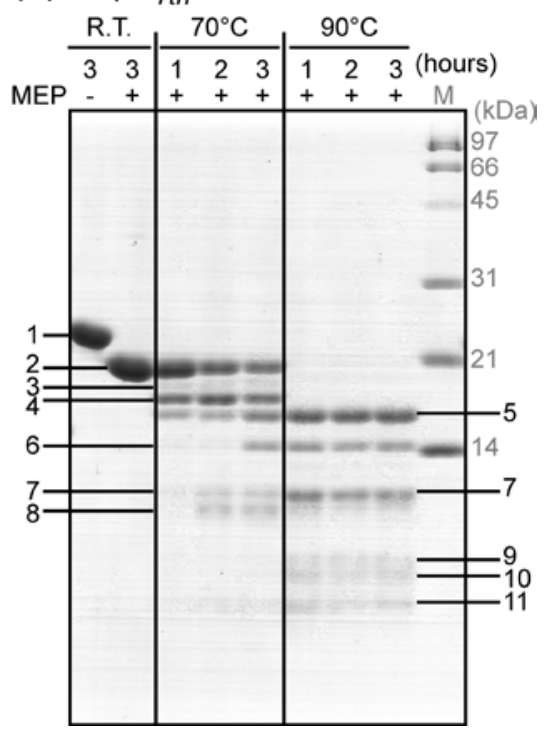

Figure 7 Nakamura et al. 
(a)

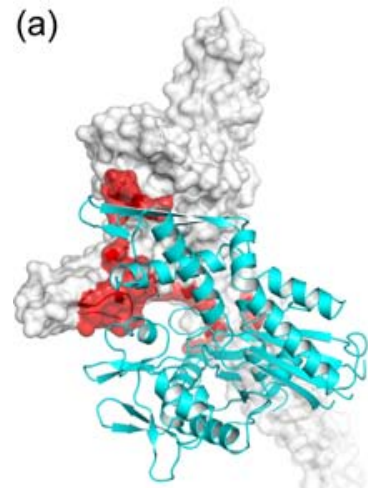

$\operatorname{GrpE}_{E c 0^{-D n a K}}$ (b)

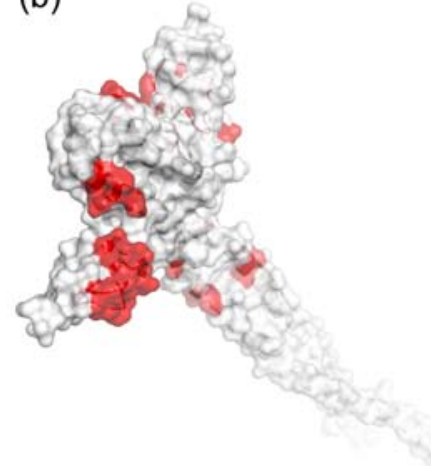

Expected DnaK binding site $\left(\mathrm{GrpE}_{T t h}\right)$ (c)

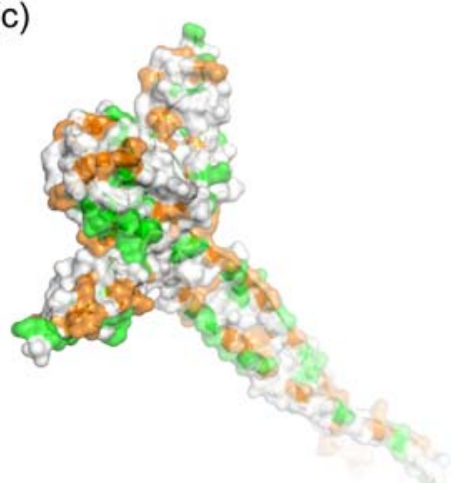

Conserved region

$\left(\mathrm{GrpE}_{T t h}\right)$

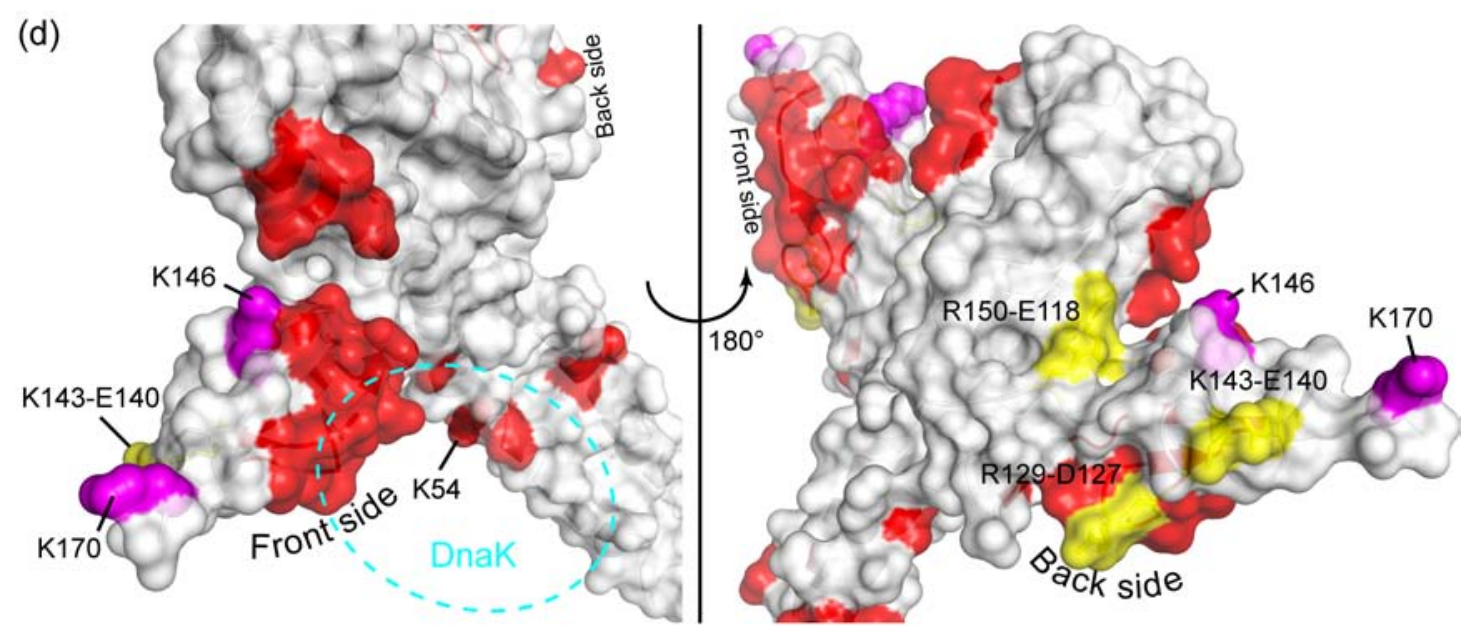

Figure 8 Nakamura et al. 


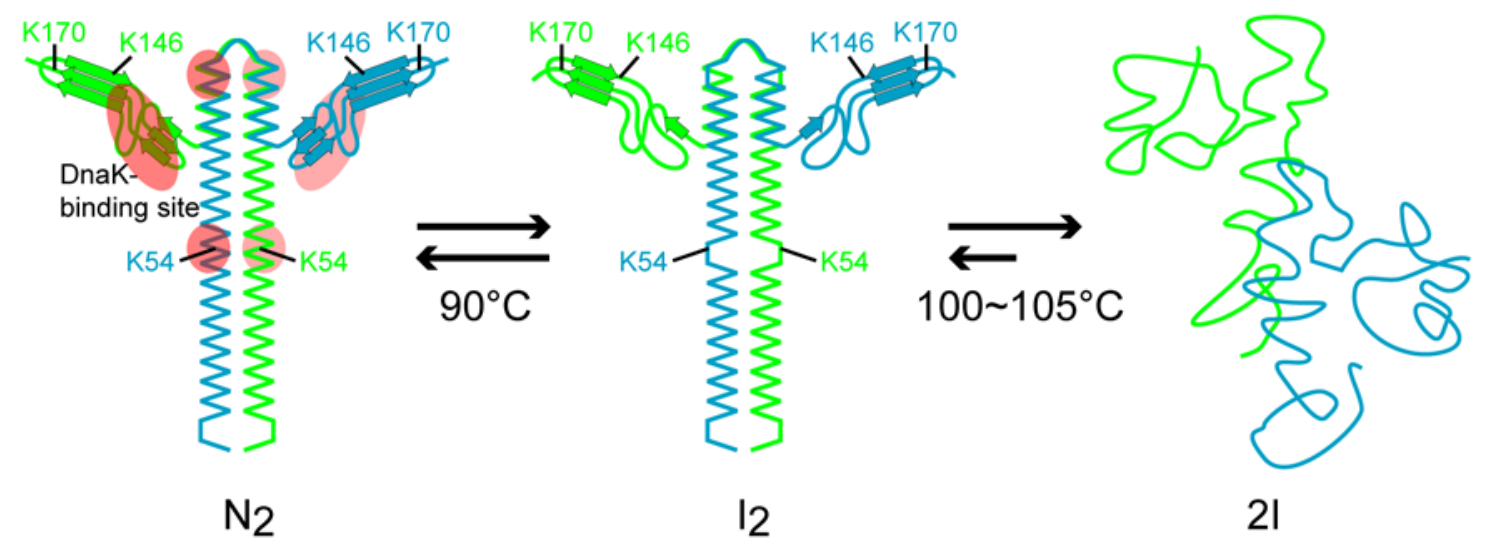

Figure 9 Nakamura et al. 